\title{
La contribución del petróleo y el carbón a la economía regional de Colombia, 1990-2011*
}

\author{
(The Contribution of Oil and Coal to the Regional Economy of \\ Colombia, 1990-2011)
}

\author{
José L. Benavides Téllez, ** M. Palma Gómez-Calero *** \\ y María del P. Pablo-Romero ****
}

\section{RESUMEN}

El objetivo de este trabajo es determinar el impacto en la economía colombiana de la producción de petróleo y carbón durante el periodo 1990-2011 y valorar de qué modo la diferente especialización en su aprovechamiento explica los desniveles del valor agregado bruto por persona ocupada observados entre las distintas regiones colombianas. Los resultados, obtenidos mediante la técnica de datos de panel, ponen de manifiesto que la dotación de petróleo y carbón contribuye positivamente a la producción colombiana y que su distinta especialización entre regiones explica $8.14 \%$ y $3.32 \%$, respectivamente, de las diferencias del valor agregado bruto por persona ocupada entre las regiones colombianas.

Palabras clave: crecimiento económico, petróleo, carbón, recursos naturales, Colombia. Clasificación JEL: L72, O47, R11.

\begin{abstract}
The aim of this paper is to determine the impact of oil and coal production on the Colombian economy for the period 1990-2011 and assess how the differences in endowments of these factors of production are explanatory of gross value added per employee differences observed between different regions in Colombia. The results obtained by the technique of panel data show that the oil and coal endowment contributes positively to Colombian production, their different endowment of oil and coal between regions explains $814 \%$ and $3.32 \%$ of the differences in gross value added per employee between the Colombian regions, respectively.
\end{abstract}

Keywords: economic growth, oil, coal, natural resources, Colombia.

JEL classification: L72, O47, R11.

* Fecha de recepción: 07/06/2013. Fecha de aprobación: 22/12/2014. Los autores agradecen la financiación recibida de la Catedra de Economía de la Energía y del Medio Ambiente.

** Consultor de desarrollo de Solimpeks Solar. Correo electrónico: jlbenavides@live.com.

*** Profesora titular del Departamento de Análisis Económico y Economía Política de la Universidad de Sevilla. Correo electrónico: mdepalma@us.es.

**** Profesora titular del Departamento de Análisis Económico y Economía Política de la Universidad de Sevilla. Correo electrónico: mpablorom@us.es. 


\section{INTRODUCCIÓN}

Desde 2004, y como ya ocurrió a comienzos del siglo XX (Ocampo, 2011), América Latina ha experimentado un importante crecimiento económico debido a la vuelta al sector primario de su estructura exportadora, con base en la explotación y exportación de recursos naturales. Esta especialización de las economías orientada a los recursos naturales es, sin embargo, intensamente discutida por los estudiosos, pues mientras algunos consideran que es positiva para la economía, otros opinan que acaba por generar un menor crecimiento.

El debate sobre este modelo adoptado por muchos países especialmente dotados de recursos naturales tiene sus orígenes en la economía clásica del siglo XVIII. Según Mideksa (2013), esta discusión se ha concentrado recientemente en el análisis del efecto que tiene para un país la dotación y explotación de recursos naturales mineros o energéticos, como el carbón o el petróleo. Entre los estudios realizados se encuentran algunos de carácter empírico que concluyen que la especialización en el aprovechamiento de recursos naturales favorece el crecimiento de los países (Weitzman, 1999; Brunnschweiler y Bulte, 2008), mientras otros llegan a resultados opuestos (Sachs y Warner, 1997; Hausman y Rigobon, 2002; Sala-I-Martin y Subramanian, 2003) reforzando la tesis de la maldición de los recursos (Auty, 1993; Murshed, 2004).

Para algunos autores, esta relación negativa está asociada a los propios factores, considerando que existe una asociación inversa entre la especialización en estos recursos y el crecimiento de la economía (Sachs y Warner, 1997). Esta relación negativa se asocia, como afirma Ocampo (2011), tanto con la llamada enfermedad holandesa como con los cambios en los precios relativos, que inducen decisiones de inversión tendientes a modificar la estructura económica, perjudicando a algunos sectores, especialmente la industria manufacturera. En este sentido, las distintas ramas productivas tienen diferente capacidad de generación del progreso técnico y también diferente capacidad de transmitir ese progreso, mediante eslabonamientos o externalidades positivas, a otras industrias o sectores para aumentar la productividad de la economía.

Para otros, la mera especialización en los recursos naturales no es negativa por sí misma, sino que la concentración de las exportaciones en estos productos es lo que puede ralentizar su economía (Lederman y Maloney, 2003). Así, en el estudio realizado por Murshed y Serino (2011), en el que se comparan economías de países con patrones de especialización comercial basados en recursos naturales, unos, y con otros que no lo están, el resultado es que las economías 
que se especializan en la producción de bienes y servicios distintos a la explotación de los recursos naturales y que diversifican su estructura exportadora obtienen un mejor crecimiento económico debido a la generación de mayor valor agregado. En este sentido, la especialización en sectores concretos puede empeorar el crecimiento, pues de acuerdo con la teoría evolucionista de Nelson y Winter (1982), los cambios técnicos en un sector pueden mejorar las técnicas de producción en el mismo, pero sin ningún, o poco, efecto de derrame en las de los sectores aledaños. Así pues, de acuerdo con Ocampo (2011), la clave del crecimiento dinámico es la sincronía entre desarrollo exportador, encadenamientos productivos y, en particular, acumulación de capacidades tecnológicas.

Por su lado, un conjunto de otros autores asocia la especialización con una gran inestabilidad política y mayor número de conflictos, que afectan negativamente al crecimiento económico; entre sus estudios cabe citar los de Mansoorian (1991), Collier, Hoeffler y Rohner (2004) y Fearon (2005). En esta misma línea, Bjorvantn, Farzanegan y Schneider (2012), en una investigación sobre países del norte de África y Medio Oriente, concluyen que el fraccionamiento del poder político es el factor que influye negativamente a la hora de que estas economías puedan beneficiarse de su riqueza petrolera.

En general, de acuerdo con Mideksa (2013), los resultados son divergentes y esta diferencia puede estar asociada a diversos problemas metodológicos, uno de los cuales, dice, es el hecho de que la mayoría de estudios están basados en análisis de datos transversales. Además, cita como uno de los problemas fundamentales, la imposibilidad de conocer a ciencia cierta lo que hubiera ocurrido en una determinada economía en ausencia de recursos naturales, pues dicha circunstancia sólo puede estimarse mediante la comparación con economías que en efecto carezcan de ellos, de las cuales existen pocas, sobre todo cuando se considera la dotación conjunta de tales recursos. Por esta razón, considera este autor, es más conveniente analizar el efecto de un solo tipo de recurso natural en un país determinado con el fin de solventar algunos de los problemas de investigaciones anteriores.

En este estudio, se propone seguir el camino marcado por Mideksa (2013) y realizar, por ello, el análisis de un recurso específico en un país concreto. De acuerdo con este enfoque, se aborda el efecto del petróleo y el carbón en la economía colombiana, aunque en lugar de analizar ese efecto sobre el total de ésta y construir un conjunto de economías comparables sin estos recursos, como hace el autor citado, se compara el efecto que tienen en las regiones de Colombia explotadoras de petróleo y carbón con respecto a aquellas que no lo son. 
Con este fin, se estima una función de producción tipo Cobb-Douglas, ampliada de acuerdo con los modelos neoclásicos de crecimiento endógenos (Romer, 1986; Lucas, 1988; Rebelo, 1991; Barro, 1991), en la que se incluye como factores explicativos de la producción la disponibilidad de capital físico, el empleo y el progreso tecnológico, que depende entre otros factores del capital humano y de la especialización en la producción de petróleo y de carbón. Esta función se estima para el conjunto de las regiones de Colombia en el periodo 1990-2011. De acuerdo con Ocampo et al. (2004), el inicio de este lapso coincide con el comienzo de la apertura económica en este país y la creación de una nueva constitución política basada en la inclusión social de todos los sectores. Por eso, este trabajo se encuadra dentro del supuesto de un mejor escenario, según la metodología utilizada por Sachs y Warner (1997).

El efecto de estos recursos naturales en la economía de Colombia ha sido analizado previamente por Perry y Olivera (2009), en un periodo comprendido entre 2000 y 2008 , desde un punto de vista regional y local. Estos autores encuentran un efecto negativo de la producción de petróleo sobre el crecimiento regional, pero positivo en el caso de la producción de carbón. No obstante, las estimaciones realizadas en su trabajo no consideran otros factores de crecimiento, como la disponibilidad de capital productivo, de empleo o de capital humano. En ese sentido, la presente investigación supone un avance al incluir estas variables explicativas del crecimiento, mediante la estimación de la función de crecimiento endógena mencionada. La exposición se hace como sigue: la primera sección describe la metodología utilizada, mientras que en la siguiente se presentan los datos de la economía colombiana utilizados en la estimación de la función de producción regional. En la tercera sección, se exponen los resultados y la última parte se ocupa de las conclusiones.

\section{MetodologíA}

Con el fin de analizar los factores determinantes del crecimiento regional en Colombia y el efecto de la producción de petróleo y carbón en tal crecimiento, se parte de una función de producción Cobb-Douglas, en la cual se incluyen como factores productivos el capital físico $(K)$, el empleo $(L)$ y una variable exógena $(A)$, que incluye otros factores determinantes de la producción, tales como el progreso tecnológico, el capital humano, las externalidades positivas, la especialización productiva o la situación geoeconómica de la región. Analíticamente, esa función se expresa del siguiente modo: 


$$
\begin{gathered}
Y_{i t}=A_{i t} K_{i t}^{\beta} L_{i t}^{\varphi} \\
A_{i t}=e^{\lambda_{t}+a_{t}+\lambda p_{i t}+\delta c_{i i}} h_{i t}^{\alpha},
\end{gathered}
$$

donde

$Y=$ valor agregado bruto

$A=$ variable exógena que incluye otros factores determinantes de la producción

$L=$ población ocupada

$K=$ disponibilidad de capital físico

$h$ = capital humano

$p$ = especialización de la región en la producción de petróleo

$c=$ especialización de la región en la producción de carbón

$i=33$ regiones de Colombia

$t=$ años, desde 1990 a 2011

$a=$ constante que muestra los efectos fijos de las regiones e incluye otros factores de la producción.

En términos logarítmicos y por persona ocupada, la expresión anterior puede escribirse así:

$$
\ln y_{i t}=\lambda_{t}+a_{i}+\gamma p_{i t}+\delta c_{i t}+\alpha \operatorname{lnh} h_{i t}+\beta \ln k_{i t}-\eta \ln L_{i t} \varepsilon_{i t}
$$

Dado que no puede rechazarse la hipótesis de rendimientos constantes de escala $(\eta=0)$ para el capital productivo y el empleo, dado que la prueba de Fisher, $F(1,720)$, da por resultado 5.13, la ecuación (1) se formula como sigue:

$$
\ln y_{i t}=\lambda_{t}+a_{i}+\gamma p_{i t}+\delta c_{i t}+\alpha \operatorname{lnh} h_{i t}+\beta \ln k_{i t} \varepsilon_{i t},
$$

donde las variables en minúsculas denotan los valores por persona ocupada. En este caso, la función se estima utilizando datos de panel para las treinta y tres regiones de Colombia durante el periodo comprendido entre 1990 y 2011, para el cual existen suficientes datos disponibles. La introducción en el modelo de efectos fijos $\left(a_{i}\right)$ se realiza con base en los resultados de la prueba de Hausman. Este econometrista demostró que la diferencia entre los coeficientes de efectos fijos y aleatorios puede ser usada para probar la hipótesis nula de que los efectos individuales y las variables exógenas no están correlacionadas. Así pues, la hipótesis 
nula (H0) de la prueba de Hausman (1978) es que los estimadores de efectos aleatorios y de efectos fijos no difieren sustancialmente. Si se rechaza la H0, los estimadores sí difieren, y la conclusión es que los efectos fijos son más convenientes que los efectos aleatorios. En nuestro caso, la diferencia entre los coeficientes de ambos puede considerarse sistemática al ser $X 2(4)=31.01$, por lo que la inclusión de modelos individuales resulta conveniente.

En primer lugar se hace la estimación por el método de mínimos cuadrados generalizados (MCG), el cual permite realizar estimaciones consistentes y más eficientes que el de mínimos cuadrados ordinarios (MCO) en presencia de heterocedasticidad y autocorrelación. El modelo de MCG, en su versión matricial, sería el siguiente:

$$
y=X \beta+u,
$$

donde $X$ hace referencia a las variables independientes del modelo y $y$ al valor agregado bruto (VAB). La matriz de varianzas y covarianzas, $\Omega$, del modelo permite cualquier tipo de estructura en los residuos sin asumir forma funcional alguna. El resultado de la minimización de estos residuos se conoce con el nombre de mínimos cuadrados generalizados, cuyos parámetros a estimar $(\gamma, \delta, \alpha \mathrm{y} \beta)$ están definidos de la siguiente manera:

$$
\beta G l s=\left(X^{\prime} \hat{\Omega}^{-1} X\right)^{-1}\left(X \hat{\Omega}^{-1} y\right),
$$

donde el estimador reemplaza las varianzas por sus estimaciones consistentes de ellas. La forma de estimar la matriz de varianzas y covarianzas depende de si la causa de su no escalaridad es la heterocedasticidad y/o la autocorrelación. En presencia de heterocedasticidad y autocorrelación, $\Omega$ queda definida en su forma general como el producto de dos matrices:

$$
\Omega-1=G^{\prime} G,
$$

quedando

$$
G=\left[\begin{array}{ccccc}
\sqrt{1-\rho^{2}} & 0 & 0 & \ldots & 0 \\
-\rho & 1 & 0 & \ldots & 0 \\
0 & -\rho & 1 & \ldots & 0 \\
\vdots & \vdots & \vdots & \vdots \\
0 & 0 & 0 & 0
\end{array}\right],
$$


donde $\rho$ es el valor estimado de la regresión de los residuos sobre los del periodo anterior.

Así pues, este método resulta adecuado cuando las varianzas de las observaciones son desiguales (presencia de heterocedasticidad) y/o cuando existe una cierta correlación entre las observaciones. La prueba de Wooldridge (2002) en datos de panel permite detectar problemas de autocorrelación. Este método utiliza los residuales de una regresión de primeras diferencias, observando que si no están correlacionados serialmente, entonces la correlación entre los errores diferenciados para el periodo $t$ y $t-1$ es igual a -0.5 . La prueba modificada de Wald para la heterocedasticidad comprueba la hipótesis nula de que no existe ningún problema de heteroscedasticidad, es decir, $\sigma_{i}^{2}=\sigma^{2}$ para toda $i=1 \ldots 33$ regiones colombianas en nuestro estudio.

La estimación del modelo no es consistente en ausencia de exogeneidad de los regresores, lo que sugiere la necesidad de modelar la variable no exógena como predeterminada para que la estimación sea insesgada. Este problema se ha tratado empleando variables instrumentales y estimando sus resultados mediante el método generalizado de momentos (MGM).

Este procedimiento consiste en realizar la estimación mediante el uso combinado de diversos instrumentos en torno a un único vector numérico de coeficientes que logre correlaciones muestrales mínimas entre el término de error y cada uno de los instrumentos. El GMM es un caso especial de estimación por variables instrumentales, en el cuál el sistema de ecuaciones e instrumentos está sobreidentificado. En esta circunstancia, para la estimación de un parámetro hay más de una restricción de momentos (condiciones de ortogonalidad).

Dicho proceso de estimación se realiza en dos etapas. En la primera se lleva a cabo una estimación de la variable que causa problemas de endogeneidad respecto a los instrumentos utilizados y al resto de covariables. El valor resultante se utiliza en la segunda etapa en lugar de la variable que producía la endogeneidad. En concreto, si se considera que la variable de capital humano causa problemas de endogeneidad y se toman como instrumentos los retardos en uno y dos periodos de dicha variable, la regresión a estimar en la primera etapa sería la siguiente:

$$
\operatorname{lnh}_{i t}=\lambda_{t}+a_{i}+\gamma p_{i t}+\delta c_{i t}+\beta \ln k_{i t+}+\alpha_{1} d \operatorname{lnh} h_{i t}+\alpha_{2} d 2 \ln h_{i t}+v_{i t},
$$

donde $d \ln h_{i t}$ y $d 2 \ln h_{i t}$ son las variables retardadas y utilizadas como instrumentos. 
En la segunda etapa, se estima la regresión

$$
\ln y_{i t}=\lambda_{t}+a_{i}+\gamma p_{i t}+\delta c_{i t}+\alpha \operatorname{lnh} h_{i t}+\beta \ln k_{i t} \varepsilon_{i t},
$$

donde $\ln h_{i t}$ son los valores resultantes de la regresión de la primera estimación.

\section{INFORMACIÓN ESTADÍSTICA}

A continuación, se reseñan las fuentes de datos empleadas para estimar la función de producción (1).

\section{Valor agregado bruto}

Los datos sobre el VAB proceden del Departamento Administrativo Nacional de Estadística (DANE, 2013a). Dicha institución es la encargada oficial de recopilar, administrar y difundir las estadísticas económicas y sociales de Colombia. Se toman los valores de la series regionales expresados en pesos colombianos (COP) constantes de 1994.

\section{Disponibilidad de capital físico}

Debido a la falta de información estadística del valor del capital físico acumulado en Colombia por regiones, este valor se ha medido indirectamente a partir de los datos de la disponibilidad de capital físico público para cada región ofrecidos por la Contaduría General de la Nacional (CGN, 2013) para el periodo de 2005 a 2011, así como de los datos suministrados por el DANE (2013a) sobre inversión anual en infraestructura, construcción de edificaciones, planta y equipo. A partir de estos datos de inversión, se obtiene una estimación retrospectiva de los valores de capital público acumulado del periodo de 1990 a 2004, con el fin de completar la serie. Posteriormente, se añaden a los datos de reservas de capital público, la inversión anual en construcciones, planta y equipo del periodo, lo que nos da un indicador de la disponibilidad de capital físico de las regiones colombianas.

Debido a la carencia de información más precisa sobre la formación bruta de capital físico por región, es imposible aproximar con mayor detalle este indicador. Los datos usados aquí no han sido ofrecidos anteriormente en ninguna publicación, lo que supone una primera estimación de su valor. 


\section{Población ocupada}

Para medir el número de personas ocupadas de la población económicamente activa, se tomó la tasa de ocupación de cada región, suministrada por el DANE (2013b), y se multiplicó por el número de personas comprendidas entre 15 y 64 años (DANE, 2013a). Sin embargo, en el caso de las regiones de Amazonia, Arauca, San Andrés, Vaupés y Vichada se utilizó el promedio de la tasa de ocupación nacional, ya que no hay datos disponibles para estas regiones.

\section{Capital humano}

También por la falta de disponibilidad de datos regionales para todo el periodo analizado, el capital humano se midió indirectamente mediante los niveles promedio de escolarización de la población. Siguiendo la metodología establecida por el Departamento Nacional de Planeación (DNP, 2013), el número de personas matriculadas en cada grado de educación primaria y secundaria de cada región, suministrado por el DANE (2013a), se dividió entre la cantidad de personas en edad de cursarlos (DANE, 2013b), es decir, la población entre cinco y 19 años. De este modo, se obtuvo un índice de escolaridad de dicha población.

Con el fin de ajustar este valor al número promedio de años de estudio, se multiplicó dicho índice por los 11 grados de primaria y secundaria sumados. El valor hallado supone una medición indirecta del número promedio de años escolares de la población, que se centra en los años de educación formal y que se ha desarrollado en trabajos empíricos como los de Mankiw, Romer y Weil, (1992); Serrano (1996); Barro y Lee (2001), y De la Fuente (2002), entre otros.

\section{Especialización en la producción de petróleo}

La especialización de una región en la producción de petróleo está determinada por el porcentaje de participación en el VAB regional, dato suministrado por el DANE (2013a). Existen regiones que no están especializadas en la producción petróleo, en cuyo caso su valor es cero.

La figura 1 muestra los valores porcentuales promedio de especialización regional en producción de petróleo para el periodo de análisis de este estudio. Las regiones especializadas son, en orden descendente: Arauca, Casanare, Meta, Putumayo, Huila, Tolima, La Guajira, Santander, Boyacá, Bolívar, Norte de Santander, Vichada, Antioquia, Cesar, Cauca, Sucre, Cundinamarca, Nariño, 
Magdalena y Córdoba. Es decir, 20 de las 33 regiones colombianas produjeron petróleo en el periodo de análisis. No obstante, en la actualidad, Magdalena no explota hidrocarburos, pues únicamente tuvo producción durante 1990 y 1991. Por su parte, Vichada sólo empezó a producir petróleo desde 2010.

Figura 1. Regiones especializadas en petróleo. Promedio porcentual de participación en el VAB regional, 1990-2011

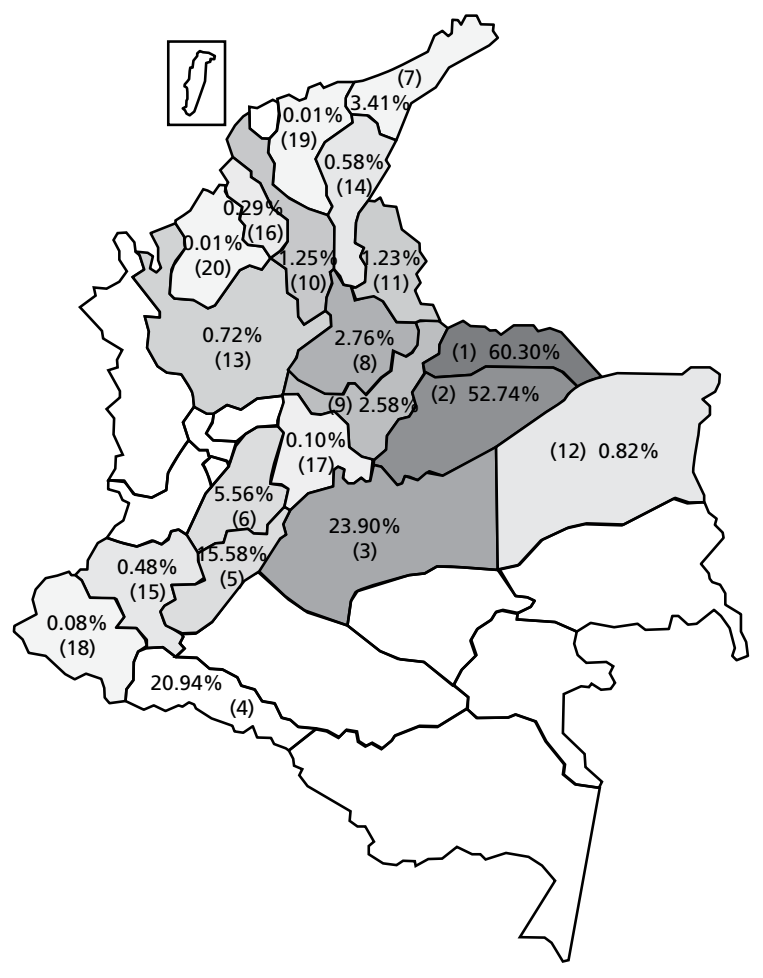

Fuente: Elaboración propia con datos del DANE (2013a).

Nota: Entre paréntesis, se indica el grado de especialización en petróleo de cada región, siendo 1 la más especializada.

\section{Especialización en la producción de carbón}

La especialización de una región en la producción de carbón está determinada por su porcentaje de participación en el VAB regional, dato proporcionado por el DANE (2013a). Existen regiones que no producen carbón, en cuyo caso el indicador toma el valor de cero. 
La figura 2 muestra los valores porcentuales promedio de especialización regional en producción de carbón para el periodo de análisis de este estudio. Las regiones especializadas en este rubro son, en orden descendente: La Guajira, Cesar, Norte de Santander, Boyacá, Cundinamarca, Córdoba, Antioquia, Valle del Cauca y Santander. Esta última empezó su extracción de carbón en el año 2000 y la región de Valle del Cauca dejó de producirlo en 2009.

Figura 2. Regiones especializadas en carbón. Promedio porcentual de participación en el VAB regional, 1990-2011

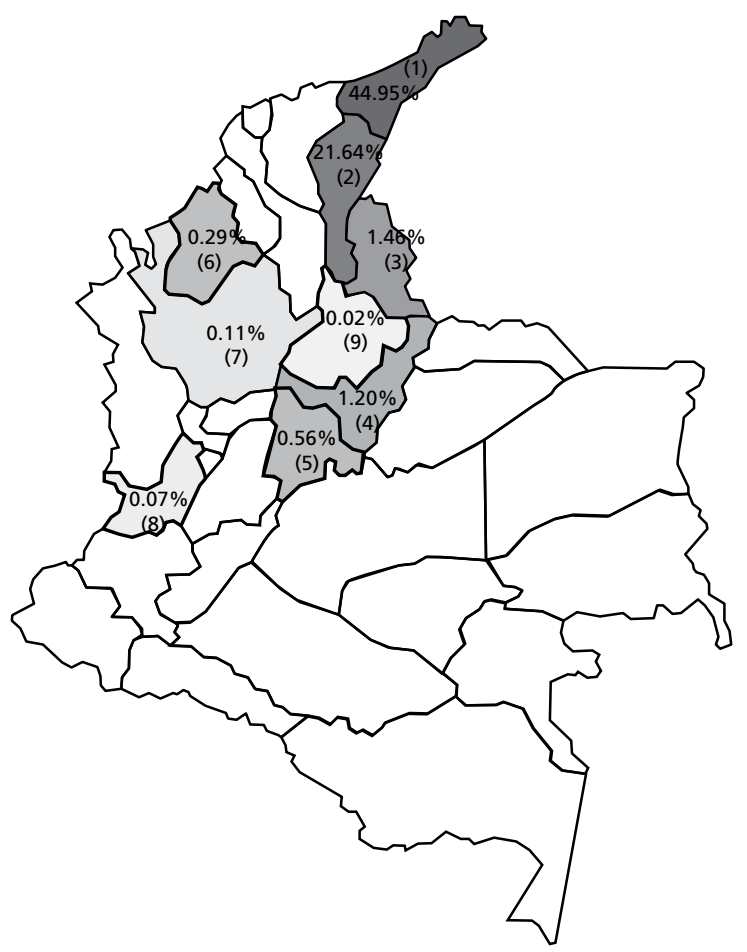

Fuente: Elaboración propia con datos del DANE (2013a).

Nota: Entre paréntesis, se indica el grado de especialización en carbón de cada región, siendo 1 la más especializada.

\section{Análisis descriptivo}

En el cuadro 1, se muestran los valores promedio de las variables descritas para cada región, en el lapso 1990-2011. Tanto las variables del VAB, como las del capital físico, están expresadas por persona ocupada. En la última fila se muestra 
Cuadro 1. VAB y factores productivos regionales por persona ocupada. Promedio de 1990-2011

\begin{tabular}{|c|c|c|c|c|c|c|}
\hline Región & $\begin{array}{c}Y \\
\text { (pesos } \\
\text { colombianos) }\end{array}$ & $\begin{array}{c}K \\
\text { (pesos } \\
\text { colombianos) }\end{array}$ & $\begin{array}{c}L \\
\text { (tasa } \\
\text { porcentual) }\end{array}$ & $\begin{array}{c}h \\
\text { (años de } \\
\text { escolaridad) }\end{array}$ & \begin{tabular}{|c|}
$p$ \\
(especialización \\
en petróleo)
\end{tabular} & $\begin{array}{c}c \\
\text { (especialización } \\
\text { en carbón) }\end{array}$ \\
\hline Amazonas & 3847182.10 & 2581518.04 & 52.17 & 5.44 & 0.00 & 0.00 \\
\hline Antioquia & 7082689.84 & 26795971.21 & 50.85 & 7.11 & 0.73 & 0.11 \\
\hline Arauca & 11232197.92 & 21427094.01 & 52.17 & 7.68 & 60.30 & 0.00 \\
\hline Atlántico & 5845032.94 & 3658695.39 & 48.73 & 6.78 & 0.00 & 0.00 \\
\hline Bogotá, DC & 7689401.49 & 13848830.06 & 57.16 & 7.70 & 0.00 & 0.00 \\
\hline Bolívar & 5846724.33 & 7304675.83 & 48.05 & 7.12 & 1.25 & 0.00 \\
\hline Boyacá & 5391095.83 & 9235489.45 & 53.17 & 6.26 & 2.59 & 1.20 \\
\hline Caldas & 6085466.29 & 8426030.67 & 49.00 & 7.86 & 0.00 & 0.00 \\
\hline Caquetá & 4638804.07 & 2426968.34 & 48.09 & 6.43 & 0.00 & 0.00 \\
\hline Casanare & 15976855.92 & 23216409.08 & 52.17 & 7.76 & 52.75 & 0.00 \\
\hline Cauca & 3193960.66 & 2565287.30 & 57.84 & 6.33 & 0.48 & 0.00 \\
\hline Cesar & 6042607.59 & 4701762.94 & 49.01 & 6.19 & 0.58 & 21.64 \\
\hline Chocó & 2818324.16 & 3696014.33 & 51.70 & 5.14 & 0.00 & 0.00 \\
\hline Córdoba & 3796758.77 & 3148038.64 & 54.42 & 7.19 & 0.01 & 0.29 \\
\hline Cundinamarca & 6022314.11 & 7423217.38 & 54.35 & 7.67 & 0.10 & 0.56 \\
\hline Guainía & 3410326.61 & 6809036.43 & 52.17 & 5.46 & 0.00 & 0.00 \\
\hline Guaviare & 8198021.11 & 5056129.72 & 52.17 & 6.39 & 0.00 & 0.00 \\
\hline Huila & 4888215.88 & 7101087.95 & 52.41 & 7.77 & 15.59 & 0.00 \\
\hline La Guajira & 5869653.97 & 9623826.44 & 50.85 & 5.37 & 3.42 & 44.95 \\
\hline Magdalena & 4220770.49 & 5110923.50 & 48.07 & 7.61 & 0.01 & 0.00 \\
\hline Meta & 6910618.90 & 6519192.05 & 56.06 & 8.57 & 23.91 & 0.00 \\
\hline Nariño & 2991704.83 & 3034294.47 & 55.28 & 7.16 & 0.08 & 0.00 \\
\hline $\begin{array}{l}\text { Norte de } \\
\text { Santander }\end{array}$ & 3922082.87 & 6282770.09 & 52.19 & 7.28 & 1.23 & 1.46 \\
\hline Putumayo & 3520579.30 & 1846457.97 & 52.17 & 5.98 & 20.94 & 0.00 \\
\hline Quindío & 4918423.06 & 12465762.64 & 48.68 & 9.16 & 0.00 & 0.00 \\
\hline Risaralda & 4994456.73 & 14401064.64 & 51.84 & 8.30 & 0.00 & 0.00 \\
\hline San Andrés & 9763067.06 & 3670086.35 & 52.17 & 5.97 & 0.00 & 0.00 \\
\hline Santander & 7084169.32 & 11326019.42 & 56.96 & 7.31 & 2.76 & 0.02 \\
\hline Sucre & 3248560.88 & 2269883.99 & 47.77 & 9.23 & 0.29 & 0.00 \\
\hline Tolima & 4735965.99 & 6470635.33 & 54.54 & 7.23 & 5.56 & 0.00 \\
\hline Valle del Cauca & 6773256.57 & 5694510.59 & 55.01 & 7.60 & 0.00 & 0.07 \\
\hline Vaupés & 4374361.26 & 3458364.93 & 52.17 & 6.80 & 0.00 & 0.00 \\
\hline Vichada & 6727767.91 & 3262672.94 & 52.17 & 7.25 & 0.83 & 0.00 \\
\hline Media global & 5820042.99 & 7722991.58 & 52.17 & 7.06 & 5.86 & 2.13 \\
\hline
\end{tabular}

Fuente: Elaboración propia, con datos del DANE (2013a; 2013b) y el CGN (2013). 
que el valor medio global del VAB por ocupado (es decir, el número trabajadores empleados para producirlo) es de COP 5820042.99 constantes de 1994. La región con un mayor VAB por ocupado es Casanare, seguida por la de Arauca. Estas dos regiones tienen una alta especialización en petróleo: $52.75 \%$ y $60.30 \%$, respectivamente.

Por otro lado, las regiones de menor VAB por ocupado son Chocó y Nariño, ya que no cuentan con especialización en los recursos analizados. El valor medio global de capital físico por persona ocupada es de COP 7222991.58 constantes de 1994. Casanare y Arauca presentan un alto nivel en este renglón, sólo por detrás de Antioquia, la cual tiene el mayor valor, mientras que las regiones con menor nivel de capital físico son Caquetá, Sucre y Putumayo, debido en parte a la falta de continuidad en la inversión en obras civiles a lo largo del periodo estudiado (DANE, 2013b). Por su parte, la tasa de ocupación oscila entre $47.77 \%$ de Sucre como valor mínimo y $57.84 \%$ de Cauca, en el otro extremo, mientras que el promedio nacional de ocupación es de $52.17 \%$.

Respecto al capital humano, el valor medio global es de 7.06 años promedio de escolaridad, observándose una gran diferencia entre los valores máximos y los mínimos regionales. Sucre presenta el mayor valor en el indicador de capital humano, con 9.23 años de escolaridad, mientras que Chocó muestra el más bajo, 5.14 años, lo que implica que la escolarización de la primera región es $44 \%$ superior a la de la segunda.

\section{RESULTADOS}

\section{Función de producción regional}

El cuadro 2 muestra la estimación de la función de producción (1) por diversos procedimientos. En la columna A se muestran los resultados de la estimación por MCG para corregir problemas de autocorrelación y heteroscedasticidad, debido a que la prueba de Wooldridge en datos de panel es $F(1,32)=42.33(0.0000)$ y la prueba modificada de Wald para heteroscedasticidad presenta un valor de $X^{2}(33)=11086.89$. La estimación del modelo no es consistente en ausencia de exogeneidad de las variables independientes. Este problema se ha tratado empleando variables instrumentales y estimando sus resultados mediante el MGM. En las columnas B, C, D y E se presentan los resultados de los coeficientes estimados y los valores de las distintas pruebas que permiten contrastar la consistencia de estimar mediante variables instrumentales en este caso. 
En la columna B se presentan los resultados de las estimaciones por MGM utilizando como instrumento la variable de capital humano, retardada en uno y dos periodos. La columna $\mathrm{C}$ agrupa los resultados al tomar el capital productivo como variable instrumental, retardada también en uno y dos niveles. Para la columna D se utilizaron los retardos del indicador de especialización de petróleo y en la E, los retardos del correspondiente al carbón. De acuerdo con lo que muestran, en la parte baja del cuadro 2, las pruebas realizadas para cada una de las estimaciones B, C, D y E, resulta más conveniente estimar por MCG, ya que los instrumentos utilizados no son suficientemente válidos, de manera que se considera más adecuada la estimación presentada en la columna A.

Cuadro 2. Estimación de la función de producción regional, 1990-2011

\begin{tabular}{|c|c|c|c|c|c|}
\hline & A & B & C & D & $\mathrm{E}$ \\
\hline & MCG & MGM (E.F) (h) & MGM (E.F) (k) & $\operatorname{MGM}(E . F)(p)$ & MGM (E.F) (c) \\
\hline $\begin{array}{l}\text { Especialización } \\
\text { en carbón }\end{array}$ & $\begin{array}{c}0.006 * * * \\
(0.001)\end{array}$ & $\begin{array}{l}0.002^{*} \\
(0.001)\end{array}$ & $\begin{array}{c}0.003 \\
(0.001)\end{array}$ & $\begin{array}{c}0.003^{* *} \\
(0.001)\end{array}$ & $\begin{array}{c}0.002 \\
(0.001)\end{array}$ \\
\hline $\begin{array}{l}\text { Especialización } \\
\text { en petróleo }\end{array}$ & $\begin{array}{l}0.005^{* * *} \\
(0.001)\end{array}$ & $\begin{array}{l}0.005^{*} \\
(0.001)\end{array}$ & $\begin{array}{l}0.005^{* * *} \\
(0.001)\end{array}$ & $\begin{array}{c}0.005^{* *} \\
(0.001)\end{array}$ & $\begin{array}{l}0.005 * * * \\
(0.001)\end{array}$ \\
\hline Capital humano & $\begin{array}{l}0.202 * * * \\
(0.052)\end{array}$ & $\begin{array}{c}0.37195 * * * \\
(0.06689)\end{array}$ & $\begin{array}{c}0.390 * * * \\
(0.066)\end{array}$ & $\begin{array}{c}0.353 * * * \\
(0.066)\end{array}$ & $\begin{array}{l}0.360 * * * \\
(0.066)\end{array}$ \\
\hline Capital físico & $\begin{array}{c}0.395 * * * \\
(0.029)\end{array}$ & $\begin{array}{c}0.20429 * * * \\
(0.04617)\end{array}$ & $\begin{array}{c}0.141 * * * \\
(0.046)\end{array}$ & $\begin{array}{c}0.211 * * * \\
(0.046)\end{array}$ & $\begin{array}{c}0.209 * * * \\
(0.046)\end{array}$ \\
\hline $\begin{array}{l}\text { Prueba F de Wald - } \\
\text { Significación conjunta }\end{array}$ & $\begin{array}{c}6.82 \mathrm{E}+ \\
04\end{array}$ & $6.20 E+05$ & $6.40 E+05$ & $6.30 E+05$ & $6.20 E+05$ \\
\hline Número de observaciones & 726 & 660 & 660 & 660 & 660 \\
\hline $\begin{array}{l}\text { Prueba de subestimación } \\
\text { de Kleibergen-Paap rk } \\
\text { LM: } X^{2}(2) \text { P-val) }\end{array}$ & & $\begin{array}{c}146.533^{* * *} \\
(0.000)\end{array}$ & $\begin{array}{c}148.975^{* * *} \\
(0.000)\end{array}$ & $\begin{array}{c}18.293 * * * \\
(0.000)\end{array}$ & $\begin{array}{l}30.717 * * * \\
(0.000)\end{array}$ \\
\hline $\begin{array}{l}\text { Prueba de debilidad del } \\
\text { estadístico Wald F de } \\
\text { Kleibergen-Paap }\end{array}$ & & 6916.738 & 2567.636 & 46.514 & 21.679 \\
\hline $\begin{array}{l}\text { Estadístico J de Hansen: } \\
\left(\mathrm{X}^{2}(1) \mathrm{P} \text {-val) }\right.\end{array}$ & & $\begin{array}{c}2.248 \\
(0.133) \\
\end{array}$ & $\begin{array}{l}12.451 * * * \\
(0.000)\end{array}$ & $\begin{array}{l}0.604 \\
(0.436)\end{array}$ & $\begin{array}{c}0.001 \\
(0.977)\end{array}$ \\
\hline $\begin{array}{l}\text { Prueba de endogeneidad } \\
\text { con regresores } \\
\text { endógenos: ( } X^{2}(1) \text { P-val) }\end{array}$ & & $\begin{array}{l}2.074 \\
(0.149)\end{array}$ & $\begin{array}{l}25.204 * * * \\
(0.000)\end{array}$ & $\begin{array}{c}0.022 \\
(0.880)\end{array}$ & $\begin{array}{c}2.513 \\
(0.112)\end{array}$ \\
\hline
\end{tabular}

Nota: Errores estándar, entre paréntesis.

$* * *<0.01 ; * *<0.05 ; *<0.10$. 
Atendiendo a los valores de dicha columna, se tienen coeficientes aproximados de 0.39 para el capital productivo, de 0.20 para el capital humano, de 0.005 para el indicador de especialización en petróleo y de 0.006 para el indicador de especialización en carbón, con un nivel de significación inferior a 15 en todas las variables. La elasticidad del capital físico es consistente con los resultados del Grupo de Estudios del Crecimiento Económico (GRECO, 2002), que halló una elasticidad, para el lapso 1950-1994, de 0.40, y con los de Villamil (2011), que reporta 0.43 .

No obstante, resulta algo inferior respecto a los de Gaviria (2005) que estima que la elasticidad de capital en Colombia fluctúa entre 0.49 y 0.67 . El resultado de la elasticidad de capital humano es semejante al obtenido por Posada (1993), que la sitúa en 0.2. Asimismo, es similar al de Gaviria (2005), ya que tras eliminar el efecto de la interacción del capital humano con el cambio técnico, la elasticidad se estima en 0.28 . No obstante, es algo inferior al reportado en el trabajo de Villamil (2011), de 0.42. El signo positivo de los indicadores respectivos señala que la especialización regional en el aprovechamiento del petróleo y el carbón influye de manera positiva en su producción por persona ocupada, es decir, en su productividad.

En este sentido, no resultan corroborados los resultados obtenidos en los trabajos previos de Bjorvantn, Farzanegan y Schneider (2012); Lederman y Maloney (2003), y Sachs y Warner (1997). Estas investigaciones se realizaron a partir de la comparación entre un conjunto de economías nacionales y dan cuenta de elasticidades negativas entre el producto interno bruto (PIB) y la producción de hidrocarburos; es por esta razón que en sus conclusiones sostienen la tesis de la maldición de los recursos naturales. Tampoco se corroboran los resultados a nivel regional obtenidos por el estudio de Perry y Olivera (2009), quienes muestran una relación negativa entre el PIB per cápita regional y la producción de petróleo, aunque cabe aclarar que en su trabajo no tienen en cuenta otros factores productivos para determinar la relación entre las variables analizadas.

Las modelos por región estiman los valores de los efectos para cada una de ellas y reflejan las circunstancias particulares que influyen en la producción regional pero que no se explican por las variables independientes consideradas. Estos valores van desde un mínimo de 8.43 para Guainía a un máximo de 9.79 para San Andrés y muestran el importante peso que tienen factores de escala, tales como el progreso tecnológico, las economías de aglomeración, la situación geoeconómica y otros que no recoge el modelo. 


\section{Diferencias de productividad entre regiones}

Los resultados obtenidos resaltan que las diferencias de productividad (medida a través del VAB por ocupado) entre regiones tienen dos orígenes: en primer lugar, una distinta especialización en cualquiera de los cuatro factores considerados $\mathrm{y}$, en segundo lugar, otras circunstancias propias de cada región que se reflejan en los distintos valores que alcanzan sus coeficientes individuales, como la situación geoeconómica, el grado de aglomeración, la estructura productiva y la capacidad de innovación.

Con el fin de analizar cuál ha sido el origen de las diferencias del VAB por ocupado entre las regiones colombianas y en qué grado incidió la especialización regional en la producción de petróleo y de carbón sobre dichas diferencias durante el periodo 1990-2011, se ha rastreado, siguiendo la metodología de Pablo-Romero y Gómez-Calero (2008), la contribución de cada factor productivo a la diferencia de productividad regional respecto a la media nacional. Es decir, se ha calculado en qué medida la especialización en cada factor productivo en una región es explicativa del diferente nivel de VAB por ocupado que se observa en las regiones colombianas.

Para ello, se han multiplicado las distintas elasticidades estimadas por el valor de la variable representativa de cada factor productivo para cada año y provincia. A continuación, se ha calculado en qué medida cada aportación por factor productivo y región difiere del correspondiente valor medio nacional para cada año. También se efectuó el mismo cálculo para el VAB por ocupado regional y el efecto fijo.

De esta manera se obtiene para cada región, año y variable un valor que puede expresarse del siguiente modo:

$$
\begin{aligned}
& y_{i t}^{0}=\ln y_{i t}-\left(\Sigma_{1}^{33} \ln y_{i t} / 33\right) \\
& c_{i t}^{0}=0.0060 \times\left[c_{i t}-\left(\Sigma_{1}^{33} c_{i t} / 33\right)\right] p_{i t}^{0}=0.0053 \times\left[p_{i t}-\left(\Sigma_{1}^{33} p_{i t} / 33\right)\right] \\
& k_{i t}^{0}=0.39 \times\left[\ln k_{i t}-\left(\Sigma_{1}^{33} \ln k_{i t} / 33\right)\right] \\
& h_{i t}^{0}=0.20 \times\left[\ln k_{i t}-\left(\Sigma_{1}^{33} \ln k_{i t} / 33\right)\right] \\
& \left.E . F_{i t}^{0}=E F_{i t}-\left(\Sigma_{1}^{33} E F_{i t} / 33\right)\right] .
\end{aligned}
$$

A partir de la diferencia entre el valor $y^{0}{ }_{i t}$ y la suma de los demás valores calculados se genera un término de error, $e_{i}$, para cada año y provincia. Para cada región se obtiene, en primer lugar, un valor que indica la diferencia de producti- 
vidad de cada una de ellas respecto a la media nacional. A continuación, se obtiene, para cada factor productivo y efecto fijo, la diferencia de las aportaciones de cada uno de éstos a la diferencia de productividad regional, así como la aportación del término de error. Estos resultados se consignan en la parte superior de cada celda del cuadro 3, de modo que el valor de la columna que corresponde al $\mathrm{VAB}$ es la diferencia de productividad de cada región respecto a la media nacional y es igual a la suma de los valores de las columnas siguientes. Por consiguiente, éstos reflejan la contribución de cada factor a la diferencia de productividad regional. A su vez, las cifras entre paréntesis muestran de qué modo la divergencia de aportación media de cada factor y efecto fijo contribuye a la diferencia del VAB por ocupado, expresado en términos porcentuales. Dada la diferencia de signos de los valores para cada región, el porcentaje respectivo no puede hallarse de forma directa, por lo cual se calcula mediante el valor absoluto de cada contribución sobre la suma de valores absolutos de las contribuciones de todos los factores, del efecto fijo y del término de error, $\mathrm{Q}_{\mathrm{i}}$. Así, por ejemplo, la contribución porcentual del capital humano para la región $i$ es igual a $\left|h_{i}^{0}\right| / Q_{i}$, siendo $Q_{i=}\left|h_{i}^{0}\right|+\left|k_{i}^{0}\right|+\left|p_{i}^{0}\right|+\left|c_{i}^{0}\right|+\left|E . F_{i}^{0}\right|+\left|e_{i}\right|$.

En la última fila del cuadro 3 , se muestran la contribución de cada factor, los efectos fijos y el error para el conjunto de la muestra. Estos resultados reflejan que la distinta disponibilidad de capital humano entre regiones colombianas explican $3.85 \%$ de la diferencia de productividad que se observa entre ellas, mientras que los desniveles de capital físico explican 40.46\%. Las distintas características específicas regionales (medidas por el efecto fijo) explican $41.96 \%$ de las diferencias de productividad y la desigual especialización en la producción de petróleo y en la de carbón explica $8.14 \%$ y 3.32\%, respectivamente.

De esta manera, se infiere que la menor o mayor dotación de recursos productivos, tales como capital físico y capital humano, repercute decisivamente en las diferencias de productividad entre las regiones, aunque la mera igualación de ellos es insuficiente para que desaparezcan las diferencias entre las regiones, pues las circunstancias específicas de cada una determinan en un alto porcentaje dichas diferencias. Esta investigación pone de manifiesto que, como parte de esas circunstancias, la especialización en la producción de petróleo y de carbón determina un claro desnivel de productividad entre las regiones.

Esto puede estar asociado, como afirma Sachs (2007), a que las ventas de la producción petrolera o carbonífera pueden ser un importante recurso para promover el desarrollo económico de la región, permitiendo la importación de bienes de capital más productivos. Asimismo, también puede asociarse a las 
62 ECONOMÍA: TEORÍA Y PRÁCTICA • Nueva Época, número 42, enero-junio 2015

Cuadro 3. Componentes de la diferencia de productividad regional respecto a su promedio, 1990-2011

(participación porcentual de cada componente en la diferencia de productividad)

\begin{tabular}{|c|c|c|c|c|c|c|c|}
\hline Región & $V A B$ & $\begin{array}{c}\text { Efectos } \\
\text { fijos }\end{array}$ & $\begin{array}{l}\text { Especialización } \\
\text { en petróleo }\end{array}$ & $\begin{array}{l}\text { Especialización } \\
\text { en carbón }\end{array}$ & $\begin{array}{l}\text { Capital } \\
\text { humano }\end{array}$ & $\begin{array}{l}\text { Capital } \\
\text { físico }\end{array}$ & Error \\
\hline & $\begin{array}{l}y^{0}{ }_{i t} \\
(100)\end{array}$ & $\begin{array}{l}E . F^{0}{ }_{i t} \\
\left(p r E F_{i}\right)\end{array}$ & $\begin{array}{c}p^{0}{ }_{i t} \\
\left(\operatorname{pr} p_{i}^{0}\right)\end{array}$ & $\begin{array}{c}c^{0}{ }_{i t} \\
\left(p r c_{i}^{0}\right)\end{array}$ & $\begin{array}{c}h_{i t}^{0} \\
\left(\operatorname{pr} h_{i}^{0}\right)\end{array}$ & $\begin{array}{c}k_{i t}^{0} \\
\left(\mathrm{pr} k_{i}^{0}\right)\end{array}$ & $\begin{array}{c}\text { Error } \\
\text { (pr error })\end{array}$ \\
\hline $\begin{array}{l}\text { Amazonas } \\
(\%)\end{array}$ & $\begin{array}{r}-0.32 \\
(100)\end{array}$ & $\begin{array}{c}0.14 \\
(22.80)\end{array}$ & $\begin{array}{l}-0.05 \\
(5.27)\end{array}$ & $\begin{array}{l}-0.03 \\
(2.14)\end{array}$ & $\begin{array}{l}-0.01 \\
(9.08)\end{array}$ & $\begin{array}{l}-0.33 \\
(55.00)\end{array}$ & $\begin{array}{l}-0.04 \\
(5.71)\end{array}$ \\
\hline $\begin{array}{l}\text { Antioquia } \\
(\%)\end{array}$ & $\begin{array}{c}0.29 \\
(100)\end{array}$ & $\begin{array}{c}-0.28 \\
(29.97)\end{array}$ & $\begin{array}{l}-0.03 \\
(2.97)\end{array}$ & $\begin{array}{l}-0.01 \\
(1.30)\end{array}$ & $\begin{array}{c}0.00 \\
(0.36)\end{array}$ & $\begin{array}{c}0.60 \\
(65.32)\end{array}$ & $\begin{array}{c}0.01 \\
(0.08)\end{array}$ \\
\hline $\begin{array}{l}\text { Arauca } \\
(\%)\end{array}$ & $\begin{array}{c}0.69 \\
(100)\end{array}$ & $\begin{array}{c}-0.13 \\
(13.04)\end{array}$ & $\begin{array}{c}0.29 \\
(30.12)\end{array}$ & $\begin{array}{l}-0.01 \\
(1.31)\end{array}$ & $\begin{array}{c}0.02 \\
(2.11)\end{array}$ & $\begin{array}{c}0.52 \\
(53.20)\end{array}$ & $\begin{array}{c}0.00 \\
(0.22)\end{array}$ \\
\hline $\begin{array}{l}\text { Atlántico } \\
(\%)\end{array}$ & $\begin{array}{c}0.10 \\
(100)\end{array}$ & $\begin{array}{c}0.35 \\
(58.02) \\
\end{array}$ & $\begin{array}{l}-0.03 \\
(5.17) \\
\end{array}$ & $\begin{array}{l}-0.01 \\
(2.10) \\
\end{array}$ & $\begin{array}{l}-0.01 \\
(0.84) \\
\end{array}$ & $\begin{array}{l}-0.20 \\
(33.53)\end{array}$ & $\begin{array}{c}0.00 \\
(0.34)\end{array}$ \\
\hline $\begin{array}{l}\text { Bogotá, DC } \\
(\%)\end{array}$ & $\begin{array}{c}0.37 \\
(100)\end{array}$ & $\begin{array}{c}0.06 \\
(12.01)\end{array}$ & $\begin{array}{l}-0.03 \\
(6.73)\end{array}$ & $\begin{array}{l}-0.01 \\
(2.74)\end{array}$ & $\begin{array}{c}0.02 \\
(4.68)\end{array}$ & $\begin{array}{c}0.34 \\
(73.44)\end{array}$ & $\begin{array}{c}0.00 \\
(0.40)\end{array}$ \\
\hline $\begin{array}{l}\text { Bolívar } \\
(\%)\end{array}$ & $\begin{array}{c}0.09 \\
(100) \\
\end{array}$ & $\begin{array}{c}0.03 \\
(18.04) \\
\end{array}$ & $\begin{array}{c}-0.02 \\
(14.91) \\
\end{array}$ & $\begin{array}{l}-0.01 \\
(7.71) \\
\end{array}$ & $\begin{array}{c}0.00 \\
(1.05)\end{array}$ & $\begin{array}{c}0.09 \\
(52.88) \\
\end{array}$ & $\begin{array}{c}0.00 \\
(5.41) \\
\end{array}$ \\
\hline $\begin{array}{l}\text { Boyacá } \\
(\%)\end{array}$ & $\begin{array}{c}0.01 \\
(100)\end{array}$ & $\begin{array}{c}-0.11 \\
(31.42)\end{array}$ & $\begin{array}{l}-0.02 \\
(5.07) \\
\end{array}$ & $\begin{array}{l}-0.01 \\
(1.61)\end{array}$ & $\begin{array}{l}-0.03 \\
(8.20)\end{array}$ & $\begin{array}{c}0.18 \\
(51.64)\end{array}$ & $\begin{array}{c}0.00 \\
(0.06)\end{array}$ \\
\hline $\begin{array}{l}\text { Caldas } \\
(\%)\end{array}$ & $\begin{array}{c}0.12 \\
(100)\end{array}$ & $\begin{array}{c}0.00 \\
(0.39)\end{array}$ & $\begin{array}{c}-0.03 \\
(14.89)\end{array}$ & $\begin{array}{l}-0.01 \\
(6.05) \\
\end{array}$ & $\begin{array}{c}0.03 \\
(12.35)\end{array}$ & $\begin{array}{c}0.14 \\
(64.80)\end{array}$ & $\begin{array}{c}0.01 \\
(1.52)\end{array}$ \\
\hline $\begin{array}{l}\text { Caquetá } \\
(\%)\end{array}$ & $\begin{array}{l}-0.14 \\
(100)\end{array}$ & $\begin{array}{c}0.32 \\
(40.88)\end{array}$ & $\begin{array}{l}-0.03 \\
(3.98)\end{array}$ & $\begin{array}{l}-0.01 \\
(1.62)\end{array}$ & $\begin{array}{l}-0.02 \\
(2.41)\end{array}$ & $\begin{array}{c}-0.40 \\
(50.76)\end{array}$ & $\begin{array}{c}0.00 \\
(0.35)\end{array}$ \\
\hline $\begin{array}{l}\text { Casanare } \\
(\%)\end{array}$ & $\begin{array}{c}1.07 \\
(100)\end{array}$ & $\begin{array}{c}0.26 \\
(23.57) \\
\end{array}$ & $\begin{array}{c}0.25 \\
(23.08)\end{array}$ & $\begin{array}{l}-0.01 \\
(1.17) \\
\end{array}$ & $\begin{array}{c}0.02 \\
(2.04) \\
\end{array}$ & $\begin{array}{c}0.53 \\
(48.58) \\
\end{array}$ & $\begin{array}{c}0.02 \\
(1.56)\end{array}$ \\
\hline $\begin{array}{l}\text { Cauca } \\
(\%)\end{array}$ & $\begin{array}{l}-0.54 \\
(100) \\
\end{array}$ & $\begin{array}{c}-0.11 \\
(21.43) \\
\end{array}$ & $\begin{array}{l}-0.03 \\
(5.39) \\
\end{array}$ & $\begin{array}{l}-0.01 \\
(2.38) \\
\end{array}$ & $\begin{array}{l}-0.02 \\
(4.18) \\
\end{array}$ & $\begin{array}{l}-0.34 \\
(64.27) \\
\end{array}$ & $\begin{array}{l}-0.03 \\
(2.35) \\
\end{array}$ \\
\hline $\begin{array}{l}\text { Cesar } \\
(\%)\end{array}$ & $\begin{array}{c}0.11 \\
(100)\end{array}$ & $\begin{array}{c}0.14 \\
(34.04) \\
\end{array}$ & $\begin{array}{l}-0.03 \\
(6.74) \\
\end{array}$ & $\begin{array}{c}0.12 \\
(27.87) \\
\end{array}$ & $\begin{array}{l}-0.03 \\
(7.78) \\
\end{array}$ & $\begin{array}{c}-0.09 \\
(22.33) \\
\end{array}$ & $\begin{array}{c}0.00 \\
(1.24) \\
\end{array}$ \\
\hline $\begin{array}{l}\text { Chocó } \\
(\%)\end{array}$ & $\begin{array}{l}-0.65 \\
(100)\end{array}$ & $\begin{array}{c}-0.32 \\
(49.93)\end{array}$ & $\begin{array}{l}-0.03 \\
(4.85)\end{array}$ & $\begin{array}{l}-0.01 \\
(1.97)\end{array}$ & $\begin{array}{c}-0.07 \\
(10.76)\end{array}$ & $\begin{array}{l}-0.19 \\
(28.66)\end{array}$ & $\begin{array}{l}-0.03 \\
(3.83)\end{array}$ \\
\hline $\begin{array}{l}\text { Córdoba } \\
(\%)\end{array}$ & $\begin{array}{l}-0.34 \\
(100)\end{array}$ & $\begin{array}{c}-0.06 \\
(14.90)\end{array}$ & $\begin{array}{l}-0.03 \\
(7.77) \\
\end{array}$ & $\begin{array}{l}-0.01 \\
(2.73) \\
\end{array}$ & $\begin{array}{c}0.00 \\
(1.04)\end{array}$ & $\begin{array}{l}-0.27 \\
(66.72)\end{array}$ & $\begin{array}{c}0.03 \\
(6.84)\end{array}$ \\
\hline $\begin{array}{l}\text { Cundinamarca } \\
(\%)\end{array}$ & $\begin{array}{l}0.13 \\
(100)\end{array}$ & $\begin{array}{c}0.05 \\
(21.42) \\
\end{array}$ & $\begin{array}{c}-0.03 \\
(14.69)\end{array}$ & $\begin{array}{l}-0.01 \\
(4.49) \\
\end{array}$ & $\begin{array}{c}0.02 \\
(9.89)\end{array}$ & $\begin{array}{c}0.10 \\
(45.64)\end{array}$ & $\begin{array}{c}0.00 \\
(3.87)\end{array}$ \\
\hline $\begin{array}{l}\text { Guainía } \\
(\%)\end{array}$ & $\begin{array}{l}-0.44 \\
(100) \\
\end{array}$ & $\begin{array}{c}-0.44 \\
(70.40) \\
\end{array}$ & $\begin{array}{l}-0.03 \\
(5.07) \\
\end{array}$ & $\begin{array}{l}-0.01 \\
(2.06) \\
\end{array}$ & $\begin{array}{l}-0.05 \\
(8.06) \\
\end{array}$ & $\begin{array}{c}0.06 \\
(10.02) \\
\end{array}$ & $\begin{array}{c}0.03 \\
(4.39) \\
\end{array}$ \\
\hline $\begin{array}{l}\text { Guaviare } \\
(\%)\end{array}$ & $\begin{array}{c}0.35 \\
(100)\end{array}$ & $\begin{array}{c}0.50 \\
(74.44)\end{array}$ & $\begin{array}{l}-0.03 \\
(4.72) \\
\end{array}$ & $\begin{array}{l}-0.01 \\
(1.92) \\
\end{array}$ & $\begin{array}{l}-0.02 \\
(2.37) \\
\end{array}$ & $\begin{array}{c}-0.10 \\
(14.39) \\
\end{array}$ & $\begin{array}{c}0.01 \\
(2.16) \\
\end{array}$ \\
\hline $\begin{array}{l}\begin{array}{l}\text { Huila } \\
(\%)\end{array} \\
\end{array}$ & $\begin{array}{l}-0.08 \\
(100) \\
\end{array}$ & $\begin{array}{c}-0.22 \\
(56.85) \\
\end{array}$ & $\begin{array}{c}0.05 \\
(13.27) \\
\end{array}$ & $\begin{array}{l}-0.01 \\
(3.25) \\
\end{array}$ & $\begin{array}{c}0.02 \\
(5.98) \\
\end{array}$ & $\begin{array}{c}0.07 \\
(16.55) \\
\end{array}$ & $\begin{array}{c}0.01 \\
(4.01) \\
\end{array}$ \\
\hline $\begin{array}{l}\text { La Guajira } \\
(\%)\end{array}$ & $\begin{array}{c}0.10 \\
(100)\end{array}$ & $\begin{array}{c}-0.31 \\
(36.39)\end{array}$ & $\begin{array}{l}-0.01 \\
(1.54)\end{array}$ & $\begin{array}{c}0.26 \\
(30.33)\end{array}$ & $\begin{array}{l}-0.05 \\
(6.41)\end{array}$ & $\begin{array}{c}0.20 \\
(23.10)\end{array}$ & $\begin{array}{c}0.01 \\
(2.23)\end{array}$ \\
\hline $\begin{array}{l}\text { Magdalena } \\
(\%)\end{array}$ & $\begin{array}{l}-0.23 \\
(100)\end{array}$ & $\begin{array}{c}-0.16 \\
(54.72) \\
\end{array}$ & $\begin{array}{c}-0.03 \\
(10.95)\end{array}$ & $\begin{array}{l}-0.01 \\
(4.46) \\
\end{array}$ & $\begin{array}{c}0.02 \\
(6.07)\end{array}$ & $\begin{array}{c}-0.06 \\
(20.60)\end{array}$ & $\begin{array}{c}0.01 \\
(3.20)\end{array}$ \\
\hline Meta & $\begin{array}{c}0.24 \\
(100)\end{array}$ & $\begin{array}{c}0.08 \\
(29.24) \\
\end{array}$ & $\begin{array}{c}0.10 \\
(33.61)\end{array}$ & $\begin{array}{l}-0.01 \\
(4.44) \\
\end{array}$ & $\begin{array}{c}0.04 \\
(15.04)\end{array}$ & $\begin{array}{c}0.04 \\
(14.01) \\
\end{array}$ & $\begin{array}{l}-0.01 \\
(3.56) \\
\end{array}$ \\
\hline
\end{tabular}




\begin{tabular}{|c|c|c|c|c|c|c|c|}
\hline Región & VAB & $\begin{array}{c}\text { Efectos } \\
\text { fijos }\end{array}$ & $\begin{array}{l}\text { Especialización } \\
\text { en petróleo }\end{array}$ & $\begin{array}{l}\text { Especialización } \\
\text { en carbón }\end{array}$ & $\begin{array}{c}\text { Capital } \\
\text { humano }\end{array}$ & $\begin{array}{l}\text { Capital } \\
\text { físico }\end{array}$ & Error \\
\hline & $\begin{array}{c}y_{i t}^{0} \\
(100)\end{array}$ & $\begin{array}{c}E . F^{0}{ }_{i t} \\
\left(p r E F_{i}\right)\end{array}$ & $\begin{array}{c}p^{0}{ }_{i t} \\
\left(\operatorname{pr} p_{i}^{0}\right)\end{array}$ & $\begin{array}{c}c^{0}{ }_{i t} \\
\left(\operatorname{prc} c_{i}^{0}\right)\end{array}$ & $\begin{array}{c}h^{0}{ }_{i t} \\
\left(\operatorname{pr} h^{0}{ }_{i}\right)\end{array}$ & $\begin{array}{c}k^{0}{ }_{i t} \\
\left(p r k_{i}^{0}\right)\end{array}$ & $\begin{array}{c}\text { Error } \\
\left(\text { pr error }_{i}\right)\end{array}$ \\
\hline $\begin{array}{l}\text { Nariño } \\
(\%)\end{array}$ & $\begin{array}{l}-0.58 \\
(100) \\
\end{array}$ & $\begin{array}{c}-0.27 \\
(44.15) \\
\end{array}$ & $\begin{array}{l}-0.03 \\
(5.03) \\
\end{array}$ & $\begin{array}{l}-0.01 \\
(2.07) \\
\end{array}$ & $\begin{array}{c}0.01 \\
(1.11) \\
\end{array}$ & $\begin{array}{c}-0.28 \\
(45.50) \\
\end{array}$ & $\begin{array}{c}0.00 \\
(2.14)\end{array}$ \\
\hline $\begin{array}{l}\text { Norte de } \\
\text { Santander } \\
(\%)\end{array}$ & $\begin{array}{l}-0.30 \\
(100) \\
\end{array}$ & $\begin{array}{c}-0.31 \\
(81.85) \\
\end{array}$ & $\begin{array}{l}-0.02 \\
(6.47) \\
\end{array}$ & $\begin{array}{c}0.00 \\
(1.04) \\
\end{array}$ & $\begin{array}{c}0.01 \\
(2.50) \\
\end{array}$ & $\begin{array}{c}0.03 \\
(7.59) \\
\end{array}$ & $\begin{array}{l}-0.01 \\
(0.55) \\
\end{array}$ \\
\hline $\begin{array}{l}\text { Putumayo } \\
(\%)\end{array}$ & $\begin{array}{l}-0.45 \\
(100) \\
\end{array}$ & $\begin{array}{c}0.01 \\
(2.13) \\
\end{array}$ & $\begin{array}{c}0.08 \\
(12.73) \\
\end{array}$ & $\begin{array}{l}-0.01 \\
(2.01) \\
\end{array}$ & $\begin{array}{l}-0.03 \\
(5.48) \\
\end{array}$ & $\begin{array}{c}-0.48 \\
(76.12) \\
\end{array}$ & $\begin{array}{l}-0.02 \\
(1.53) \\
\end{array}$ \\
\hline $\begin{array}{l}\text { Quindío } \\
(\%)\end{array}$ & $\begin{array}{l}-0.08 \\
(100)\end{array}$ & $\begin{array}{c}-0.40 \\
(49.18) \\
\end{array}$ & $\begin{array}{l}-0.03 \\
(3.85) \\
\end{array}$ & $\begin{array}{l}-0.01 \\
(1.56) \\
\end{array}$ & $\begin{array}{c}0.06 \\
(6.97) \\
\end{array}$ & $\begin{array}{c}0.30 \\
(36.19) \\
\end{array}$ & $\begin{array}{c}0.00 \\
(2.25) \\
\end{array}$ \\
\hline $\begin{array}{l}\text { Risaralda } \\
(\%)\end{array}$ & $\begin{array}{c}-0.06 \\
(100)\end{array}$ & $\begin{array}{c}-0.42 \\
(48.53)\end{array}$ & $\begin{array}{l}-0.03 \\
(3.67)\end{array}$ & $\begin{array}{l}-0.01 \\
(1.49)\end{array}$ & $\begin{array}{c}0.04 \\
(4.33)\end{array}$ & $\begin{array}{c}0.36 \\
(41.69)\end{array}$ & $\begin{array}{c}0.00 \\
(0.29)\end{array}$ \\
\hline $\begin{array}{l}\text { San Andrés } \\
(\%)\end{array}$ & $\begin{array}{c}0.61 \\
(100) \\
\end{array}$ & $\begin{array}{c}0.92 \\
(74.95) \\
\end{array}$ & $\begin{array}{l}-0.03 \\
(2.57) \\
\end{array}$ & $\begin{array}{l}-0.01 \\
(1.04) \\
\end{array}$ & $\begin{array}{l}-0.03 \\
(2.45) \\
\end{array}$ & $\begin{array}{c}-0.21 \\
(17.12) \\
\end{array}$ & $\begin{array}{l}-0.03 \\
(1.87) \\
\end{array}$ \\
\hline $\begin{array}{l}\text { Santander } \\
(\%)\end{array}$ & $\begin{array}{c}0.27 \\
(100)\end{array}$ & $\begin{array}{c}0.02 \\
(6.73)\end{array}$ & $\begin{array}{l}-0.02 \\
(5.07) \\
\end{array}$ & $\begin{array}{l}-0.01 \\
(3.86)\end{array}$ & $\begin{array}{c}0.01 \\
(3.19) \\
\end{array}$ & $\begin{array}{c}0.26 \\
(78.26) \\
\end{array}$ & $\begin{array}{c}0.01 \\
(2.89) \\
\end{array}$ \\
\hline $\begin{array}{l}\text { Sucre } \\
(\%)\end{array}$ & $\begin{array}{l}-0.49 \\
(100) \\
\end{array}$ & $\begin{array}{c}-0.12 \\
(18.91) \\
\end{array}$ & $\begin{array}{l}-0.03 \\
(4.68) \\
\end{array}$ & $\begin{array}{l}-0.01 \\
(2.00) \\
\end{array}$ & $\begin{array}{c}0.06 \\
(9.18) \\
\end{array}$ & $\begin{array}{c}-0.40 \\
(63.15) \\
\end{array}$ & $\begin{array}{c}0.01 \\
(2.08) \\
\end{array}$ \\
\hline $\begin{array}{l}\text { Tolima } \\
(\%)\end{array}$ & $\begin{array}{l}-0.11 \\
(100)\end{array}$ & $\begin{array}{c}-0.16 \\
(68.18) \\
\end{array}$ & $\begin{array}{c}0.00 \\
(0.69) \\
\end{array}$ & $\begin{array}{l}-0.01 \\
(5.51) \\
\end{array}$ & $\begin{array}{c}0.01 \\
(3.37) \\
\end{array}$ & $\begin{array}{c}0.03 \\
(14.83) \\
\end{array}$ & $\begin{array}{c}0.02 \\
(7.42)\end{array}$ \\
\hline $\begin{array}{l}\text { Valle } \\
(\%)\end{array}$ & $\begin{array}{c}0.25 \\
(100) \\
\end{array}$ & $\begin{array}{c}0.28 \\
(77.18) \\
\end{array}$ & $\begin{array}{l}-0.03 \\
(8.54) \\
\end{array}$ & $\begin{array}{l}-0.01 \\
(3.36) \\
\end{array}$ & $\begin{array}{c}0.02 \\
(5.15) \\
\end{array}$ & $\begin{array}{l}-0.02 \\
(4.76) \\
\end{array}$ & $\begin{array}{c}0.01 \\
(1.01)\end{array}$ \\
\hline $\begin{array}{l}\text { Vaupés } \\
(\%)\end{array}$ & $\begin{array}{l}-0.19 \\
(100) \\
\end{array}$ & $\begin{array}{c}0.08 \\
(21.25) \\
\end{array}$ & $\begin{array}{l}-0.03 \\
(7.93) \\
\end{array}$ & $\begin{array}{l}-0.01 \\
(3.22) \\
\end{array}$ & $\begin{array}{c}0.00 \\
(1.24) \\
\end{array}$ & $\begin{array}{c}-0.25 \\
(62.09) \\
\end{array}$ & $\begin{array}{c}0.02 \\
(4.27) \\
\end{array}$ \\
\hline $\begin{array}{l}\text { Vichada } \\
(\%)\end{array}$ & $\begin{array}{c}0.21 \\
(100) \\
\end{array}$ & $\begin{array}{c}0.58 \\
(59.74) \\
\end{array}$ & $\begin{array}{l}-0.03 \\
(2.79) \\
\end{array}$ & $\begin{array}{l}-0.01 \\
(1.32) \\
\end{array}$ & $\begin{array}{c}0.01 \\
(0.99) \\
\end{array}$ & $\begin{array}{c}-0.30 \\
(31.32) \\
\end{array}$ & $\begin{array}{l}-0.04 \\
(3.84) \\
\end{array}$ \\
\hline$(\mathrm{t})$ & 44.25 & 10.71 & 5.35 & 2.59 & 7.23 & 11.90 & 6.42 \\
\hline (\%) Global & $(100)$ & $(41.96)$ & (8.14) & (3.32) & (3.85) & $(40.46)$ & $(2.27)$ \\
\hline
\end{tabular}

Número de observaciones: 396.

ventajas que conllevan las externalidades positivas que estas industrias pueden generar, al permitir un mayor desarrollo de economías de aglomeración. Asimismo, el cuadro 3 muestra que la diferencia de productividad en el caso de las regiones que cuentan con una especialización petrolífera más acusada (Arauca, Casanare y Meta) se explica en 30.08\%, 23.08\% y 33.61\%, respectivamente debido precisamente a esa especialización. Estas regiones tienen un VAB por ocupado que supera la media nacional, siendo Meta la región con mayor diferencia de productividad.

Por su lado, Putumayo y Huila, aunque están especializadas en producción de petróleo, muestran una productividad inferior a la media. Esta diferencia se explica, en el caso de Putumayo, en $76.12 \%$ por la escasa dotación de capital físico con que cuenta. En cambio, para la región de Huila, se explica en 56.85\% 
por sus características individuales recogidas en los efectos fijos, que están por debajo de la media nacional.

Asimismo, se observa que La Guajira y Cesar tienen una productividad superior a la media nacional, lo que se explica en $30.33 \%$ y $27.87 \%$, respectivamente, por su especialización en la producción de carbón. Otro factor que explica en $23.10 \%$ el alto nivel de productividad de La Guajira es su capital físico. En Cesar, además de la importante especialización en producción carbonífera, sus características individuales explican en $34.04 \%$ su diferencial positivo de productividad.

\section{Crecimiento de la productividad regional}

Con el fin de completar este análisis, se establece la contribución de cada factor productivo al crecimiento regional en el periodo 1990-2011. Dicha contribución se obtiene multiplicando las tasas de crecimiento de cada factor por región $\left(\Delta \mathrm{p}_{i p^{\prime}} \Delta \mathrm{c}_{i i^{\prime}} \Delta k_{i p^{\prime}} \Delta h_{i t}\right)$ por los coeficientes estimados de la función (1) $\left(\gamma \Delta \mathrm{p}_{i p^{\prime}} \delta \Delta \mathrm{c}_{i p^{p}}\right.$ $\left.\beta \Delta k_{i{ }^{\prime}} \alpha \Delta h_{i t}\right)$. Mediante la suma de estas contribuciones se halla la productividad total de los factores (PTF) a modo de residuo de Solow. El valor porcentual de la contribución de cada factor y la PTF al crecimiento del VAB por ocupado se calcula, para reducir el peso de las estimaciones atípicas, mediante una regresión que toma la forma $c p_{i t}=a Q_{i t}+u_{i t}$ en el caso de la especialización petrolífera y otras equivalentes para cada uno de los demás factores, siendo $Q_{i t}=\left|\gamma \Delta p_{i t}\right|+\left|\delta \Delta c_{i t}\right|+\mid$ $\beta \Delta k_{i t}|+| \alpha \Delta h_{i t}|+| P T F_{i t} \mid$.

Cuadro 4. Contribución al crecimiento regional de Colombia por componente, 1990-2011

\begin{tabular}{lcccccc}
\hline & $\begin{array}{c}\text { Tasa de } \\
\text { crecimiento } \\
\text { global }\end{array}$ & $\begin{array}{c}\text { Especialización } \\
\text { en petróleo } \\
\text { a }\end{array}$ & $\begin{array}{c}\text { Especialización } \\
\text { en carbón } \\
\text { b }\end{array}$ & $\begin{array}{c}\text { Capital } \\
\text { humano } \\
\text { c }\end{array}$ & $\begin{array}{c}\text { Capital } \\
\text { físico } \\
\text { d }\end{array}$ & $\begin{array}{c}\text { PTF } \\
\text { E }\end{array}$ \\
\hline Proporción global & 100 & 21.52 & 0.22 & 4.59 & 23.98 & 49.61 \\
(t) & & $(5.32)$ & $(2.40)$ & $(4.45)$ & $(4.38)$ & $(116.46)$ \\
\hline
\end{tabular}

Número de observaciones: 198.

Estos valores porcentuales se muestran en el cuadro 4. De acuerdo con ellos, el crecimiento de la productividad para el conjunto de las regiones colombianas queda explicado en $21.52 \%$ por el incremento de la especialización en la 
producción de petróleo, en $0.22 \%$ por la mayor especialización en carbón, en $4.59 \%$ por el capital humano, en $23.98 \%$ por el capital físico y, finalmente, en $49.61 \%$ por la PTF.

\section{CONCLUSIONES}

En este trabajo se analiza de qué modo la especialización en la producción de petróleo y de carbón ha contribuido a la producción regional de Colombia desde 1990 hasta 2011. Para ello, se estimó una función de producción con rendimientos constantes a escala considerada en niveles por el método de mínimos cuadrados generalizados.

Los coeficientes obtenidos para los factores utilizados en la función son: 0.0053 para la especialización en petróleo, 0.0060 para la especialización en carbón, 0.20 para el capital humano y 0.39 para el capital físico. Los respectivos signos positivos en los casos de la especialización en petróleo y en carbón determinan que la explotación de estas materias primas beneficia la productividad de las regiones especializadas en ello. En este sentido, no se corroboran los resultados obtenidos por Sachs y Warner (1997); Lederman y Maloney (2003), Bjorvantn, Farzanegan y Schneider (2012) y Perry y Olivera (2009), los cuales reportan elasticidades negativas entre el VAB y la producción de hidrocarburos, sosteniendo la tesis de la maldición de los recursos naturales.

Las regiones de Casanare, Arauca y Meta cuentan con los valores más altos de especialización en petróleo y es este factor el que explica en $23.08 \%$, $30.12 \%$ y 33.61 , respectivamente, su diferencia en productividad, que tiene un valor superior al promedio nacional, al igual que su capital humano y su capital físico. La Guajira y Cesar son las regiones más especializadas en producción carbonífera, lo que explica en $30.33 \%$ y $27.87 \%$, respectivamente, su diferencia en productividad, que también es superior al promedio nacional. La región de Putumayo cuenta con una especialización de $21 \%$ en producción de petróleo. Dicho factor explica en $13 \%$ su diferencia en productividad, sin embargo, ésta se encuentra por debajo del promedio nacional, debido a su escasa dotación de capital humano y de capital físico.

En términos globales, la distinta especialización en producción petróleo de las regiones explica en $8.14 \%$ la diferencia de productividad entre ellas, mientras que el desnivel en especialización carbonífera lo hace en $3.32 \%$, el de capital humano en $3.85 \%$, el de capital físico en $40.21 \%$ y las particulares características regionales en $41.96 \%$. Con estos resultados, se puede concluir que la menor 
especialización en petróleo y en carbón repercute significativamente en las diferencias de productividad de cada región.

El crecimiento de la productividad para el conjunto de las regiones colombianas queda explicado en $21.52 \%$ por la especialización en petróleo, en $0.22 \%$ por la especialización en carbón, en $23.98 \%$ por el capital físico, en $4.59 \%$ por el capital humano y en $49.61 \%$ por la PTF. En futuras investigaciones puede resultar conveniente realizar análisis sobre las relaciones entre factores productivos y su contribución al crecimiento. En este sentido, la estimación con funciones trascendentes logarítmicas puede ser conveniente.

\section{REFERENCIAS BIBLIOGRÁFICAS}

Auty, Richard (1993), Sustaining Development in Mineral economies: the resource curse thesis, Londres, Routledge.

Barro, Robert (1991), "Economics growth in a cross section of countries", Quaterly Journal of Economies, 106 (2), pp. 407-444.

Barro, Robert, y Lee, Jong-Wha (2001), "Internacional measures of educational attainement updates and implications", Oxford Economics Papers, 53 (3), pp. 541-563.

Bjorvantn, Kjetil; Farzanegan, Mohammad, y Schneider, Friedrich (2012), "Resource curse and power balance: Evidence from oil-rich countries", World Development, 40 (7), pp. 1308-1316.

Brunnschweiler, Christa, y Bulte, Erwin (2008), “The resource curse revisited and revised: a tale of paradoxes and red herrings", Journal of Environmental Economics and Management, 55 (3), pp. 248-264.

CGN (2013), "Series históricas", en Contaduría General de la Nación. Consultado el 19 de diciembre de 2014, en: www.contaduria.gov.co/wps/portal/internetes/home/ internet/inicio/!ut/p/b1/04/

Collier, Paul; Hoeffler, Ankem, y Rohner, Dominic (2004), "Greed and grievance in civil war", Oxford Economic Papers, 56 (4), pp. 563-595.

DANE (2013a), "Cuentas nacionales, económicas y sociales", en Departamento Administrativo Nacional de Estadística. Consultado el 19 de diciembre de 2014, en: www.dane.gov.co/index.php?option=com_content\&view=article\&id=99\&Item id $=74$

DANE (2013b), "Informe de coyuntura económica regional-ICER-2011", en Departamento Administrativo Nacional de Estadística. Consultado el 19 de diciembre de 2014, en: www.dane.gov.co/index.php?option=com_content\&view=article\&id= 99\&Itemid $=74$ 
DNP (2013), "La educación en cifras", en Departamento Nacional de Planeación. Consultado el 19 de diciembre de 2014, en: www.dnp.gov.co/Portals/0/archivos/documentos/DDS/SISD/boletin33.pdf

Fearon, James (2005), "Primary commodity exports and civil war", Journal of Conflict Resolution, 49 (agosto), pp. 483-506.

Fuente, Ángel de la (2002), "Capital humano y crecimiento: nuevas series de escolarización y algunos resultados para la OCDE”, Economía Industrial, 0 (348), pp. 41-52.

Gaviria, Mario (2005), “Comercio exterior, capital humano y crecimiento económico en Colombia", tesis de Maestría en Ciencias Económicas, Universidad Nacional de Colombia, sede Medellín.

GRECO (2002), El crecimiento económico colombiano en el siglo XX, Bogotá, Fondo de Cultura Económica.

Hausman, Jerry A. (1978), "Specification Tests in Econometrics", Econometrica, 46 (6), pp. 1251-1271.

Hausman, Ricardo, y Rigobon, Roberto (2002), “An Alternative Interpretation of the Resource Curse: Theory and Policy Implications", NBER Working Paper Series 9424.

Lederman, Daniel, y Maloney, William (2003), Trade Structure and Growth, Washington, DC, Office of the Chief Economist for Latin America and the Caribbean/ The World Bank.

Lucas, Robert (1988), "On the mechanics of economic development”, Journal of Monetary Economics, 22 (1), pp. 3-42.

Mankiw, Gregory; Romer, David, y Weil, David (1992), “A contribution to the empirics of economic growth”, Quaterly Journal of Economics, 107 (2), pp. 407-437.

Mansoorian, Arman (1991), "Resource discoveries and excessive external borrowing", Economic Journal, 101 (409), pp. 1497-1509.

Mideksa, Torben (2013), "The economic impact of natural resources", Journal of Environmental Economics and Management, 65 (2), pp. 277-289.

Murshed, Syed (2004), "When does natural resource abundance lead to a resource curse?”, EEP Discussion Paper 04-01, International Institute for Environment and

Development, Londres.

Murshed, Syed, y Serino, Leandro (2011), "The pattern of specialization and economic growth: The resource curse hypothesis revisited", Structural Change and Economic Dynamics, 22 (2), pp. 151-161.

Nelson, Richard, y Winter, Sidney (1982), An Evolutionary Theory of Economic Change, Cambridge, Belknap Press/Harvard University Press.

Ocampo, José (2011), “El auge de los precios de productos básicos y el riesgo de enfer- 
medad holandesa en América Latina”, Boletín Informativo Techint, 0 (336), pp. $25-48$.

Ocampo, José; Sánchez, Fabio; Hernández, Gustavo, y Prada, María (2004), Crecimiento de las exportaciones y sus efectos sobre el empleo, la desigualdad y la pobreza en Colombia, Bogotá, DC, Universidad de los Andes.

Pablo-Romero, María, y Gómez-Calero, María (2008), “Análisis por provincias de la contribución del capital humano a la producción en la década de los noventa", Investigaciones Económicas, 32 (1), pp. 27-52.

Perry, Guillermo, y Olivera, Mauricio (2009), "El impacto del petróleo y la minería en el desarrollo regional y local en Colombia", CAF Documentos de Trabajo 2009/06.

Posada, Carlos (1993), “Crecimiento económico, capital humano y educación: la teoría y el caso colombiano posterior a 1945”, Revista Planeación y Desarrollo, 24 (1), pp. 1-23.

Rebelo, Sergio (1991), “Long run policy analysis and long run growth", Journal of Political Economy, 99 (3), pp. 500-521.

Romer, Paul (1986), "Increasing returns and long run growth", Journal of Political Economy, 94 (5), pp. 1002-1037.

Sachs, Jeffrey (2007), "How to handle the macroeconomics of oil wealth", en M. Humphreys, J. Sachs y J. Stiglitz (eds.), Escaping the Resource Curse, New York, Columbia University Press, pp. 173-193.

Sachs, Jeffrey, y Warner, Andrew (1997), Natural resource abundance and economic growth, Cambridge, Harvard University.

Sala-I-Martin, Xavier, y Subramanian, Arvind (2003), “Addressing the natural resource curse: An illustration from Nigeria”, IMF Working Paper WP/03/139.

Serrano, Lorenzo (1996), "Indicadores de capital humano y productividad", Revista de Economía Aplicada, 4 (10), pp. 177-190.

Solow, Robert (1956), "A contribution to the theory of economic growth", The Quaterly Journal of economics, 70 (1), pp. 65-94.

Villamil, Héctor (2011), "El capital humano como impulsor del crecimiento económico en Colombia", Administración \& Desarrollo, 39 (54), pp. 151-166.

Wooldridge, Jeffrey (2002), Econometric Analysis of Cross Section and Panel Data, Cambridge, MIT Press.

Weitzman, Martin (1999), "Pricing the limits to growth from minerals depletion", Quarterly Journal of Economics, 114 (2), pp. 691-706. 